\title{
An Improved Denoising Method of Structured Light Image Based on Wavelet Transform
}

\author{
Gan Liu ${ }^{a,{ }^{*}}$, Xinjie Shao ${ }^{b}$ \\ Department of Vehicles and Electrical Engineering, Ordance Engineering College, No.97 Heping \\ West Road, Shijiazhuang, Hebei, China \\ a819249800@qq.com, bshaoxinjie2000@163.com
}

Keywords: image processing, structured light, median filter, wavelet transforms.

\begin{abstract}
The structured light stripe images are usually subjected to a lot of noise interference, which will affect the analysis of the image, such as: the center of light stripe extraction and edge detection. In this paper, a denoising method using adaptive median filter and improved wavelet reconstruction is proposed, which is on basis of the analysis of the noise of structured light stripe images and characteristics of the two methods. The proposed method is used to denoise the structured light stripe image, and the results are compared with the traditional wavelet soft threshold denoising method and any another denoising methods. The objective evaluation results show that the denoising method proposed in this paper has better denoising effect on structured light stripe images.
\end{abstract}

\section{Introduction}

The processing of light stripe image is an important step in the detection technology of structured light vision [1]. Due to the light source, environmental noise and the measured surface characteristics and other factors, the laser stripe image acquired contain a large number of noise [2], It will bring greater uncertainty to the results of subsequent image processing, so it is necessary to denoise the light stripe image.

In this paper, through analyzing the characteristics of the structured light image noise, combined with the adaptive median filter and improved wavelet image denoising algorithm, the wavelet denoising effect of the image improved, it is beneficial to the accuracy of the further analysis of the image.

\section{Analysis of Structured Light Image Noise}

\subsection{The Source of Image Noise}

The sources of structured light image noise are mainly two aspects: first is the measurement systems, the second is the measured object surface reflection characteristics.

1. Some components of structured light measurement system may be the source of the noise, such as: power supply, amplifier and the CCD sensor, etc. When the system is working, the current will produce the shot noise and thermal noise, the photons will produce quantum noise. The shot noise and thermal noise are in accordance with the Gauss distribution, which is additive noise, and the quantum noise in accordance with Poisson distribution, which is not additive noise [3].

2. The incident light is scattered on the surface of the object because of the material of the surface is not even, part of the scattered light is mirror reflection light, which follows the law of geometrical optics reflection. Another part of the scattered light is reflected in all directions of the space, which leads to the uncertainty of the light intensity values of the structured light stripe collected by the system [4].

\subsection{Effect of Noise on Line Structured Light Stripe}

Under ideal conditions, the cross section intensity distribution of line structured light stripe is close to the Gauss distribution [1], as shown in figure 1. In fact, due to the interference of noise and the characteristics of the surface of the object, the light intensity distribution of the actual structure is 
far from the ideal distribution, as shown in figure 1 . So the analysis accuracy of the structured light stripe image can be reduced, such as: centerline extraction, edge extraction, etc.
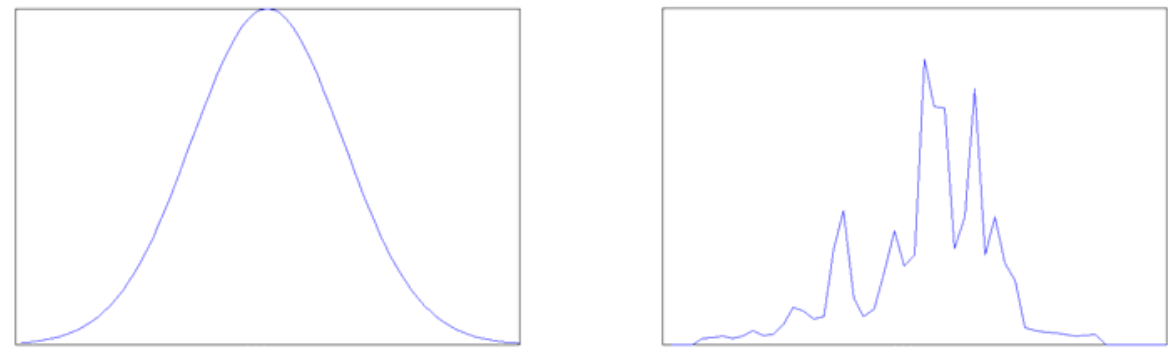

Fig. 1 Cross section intensity distribution of structured light

stripe under idea conditions and actual situations

\section{Denoising Principle}

\subsection{Adaptive Median Filtering}

Median filtering is a widely used nonlinear filtering technique was used most widely based on scheduling theory, especially has a good effect on the removal of impulse noise, and can protect the edge of the image, to avoid the phenomenon of fuzzy linear filter to the image. The principle of median filter is to sort the pixel gray value of each pixel in the neighborhood and the median is used as the gray value of the pixel after filtering. The neighborhood window moves along the lines and columns of the digital image to complete the filtering of the whole image ${ }^{[5]}$.

Adaptive median filter is an improved filtering algorithm based on median filtering:

1. Set a variable filter window with a maximum size $(2 n+1)^{*}(2 n+1)$. Firstly, the median filter value Imin, maximum filter value Imax and minimum filter value Imin of image pixels is calculated by using $3 * 3$ rectangular filter window, Then determine whether Imed is between Imin and Imax, if yes, step2, if not, then gradually increase the size of the filter window until the maximum size of the filter window

2. If the current filter pixel gray value I(x,y) between Imin and Imax , output current pixel value. Otherwise, output Imed.

\subsection{Principle of Wavelet Transform Denoising}

Wavelet transform is a signal processing technique based on multiresolution analysis, it is the extension of one dimensional signal wavelet transform to two-dimensional image. Image wavelet transform has been widely used in many fields such as image denoising, image enhancement, image fusion and so on.

Wavelet transform is a signal processing technique based on multiresolution analysis, it is the extension of one dimensional signal wavelet transform to two-dimensional image. Image wavelet transform has been widely used in many fields such as image denoising, image enhancement, image fusion and so on.

The essence of wavelet transform is to perform wavelet transform in the direction of line and column of the image independently. First, perform wavelet transform in the direction of horizontal to obtain two sub images with horizontal resolution reduced by half, which are low frequency components and high frequency components in the direction of vertical. Then the wavelet transform is performed on the vertical direction to obtain low frequency and high frequency components of two sub images in the horizontal direction. Finally, we get four sub images with size of 1/4 original image which corresponding to the low frequency components of the image, the high frequency components in the vertical direction of the image, the high frequency components in the horizontal direction of the image, and the high frequency components on the diagonal lines of the image. If the low-frequency components continue to carry out the above process, we can get the multilevel wavelet decomposition of the original image. 
The inverse process of wavelet transform is the process of image reconstruction, before the reconstruction of the image, the high frequency components of the image are subjected to threshold quantization processing, and then reconstructs the image signal by wavelet, if the original signal is a noisy signal, the reconstructed signal is denoised image. This image denoising method is the wavelet threshold denoising method. This method is not sensitive to Gauss noise in images, and can preserve the image details.

\subsection{Image Denoising Method Based on Adaptive Median Filter and Wavelet Transform}

The adaptive median filter can remove the impulse noise and preserve the details of the original image in the image, and the wavelet transform denoising has a better filtering effect of Gauss noise, this paper uses the combined method of structured light image filtering. According to the analysis of the noise in the image, the adaptive median filter is used to preprocess the original image. Then the result image is decomposed by wavelet transform, and the low-frequency part of the decomposition is not processed, median filtering in vertical direction is performed on the high-frequency components in the vertical direction, median filtering in horizontal direction is performed on the high-frequency components in the horizontal direction, median filtering in diagonal direction is performed on the high-frequency components in the diagonal direction, The wavelet coefficients are reconstructed by the high frequency coefficients obtained by directional median filtering and the low frequency coefficients obtained by the original wavelet decomposition, thus the denoised image is obtained. Specific algorithm flow is as follows:

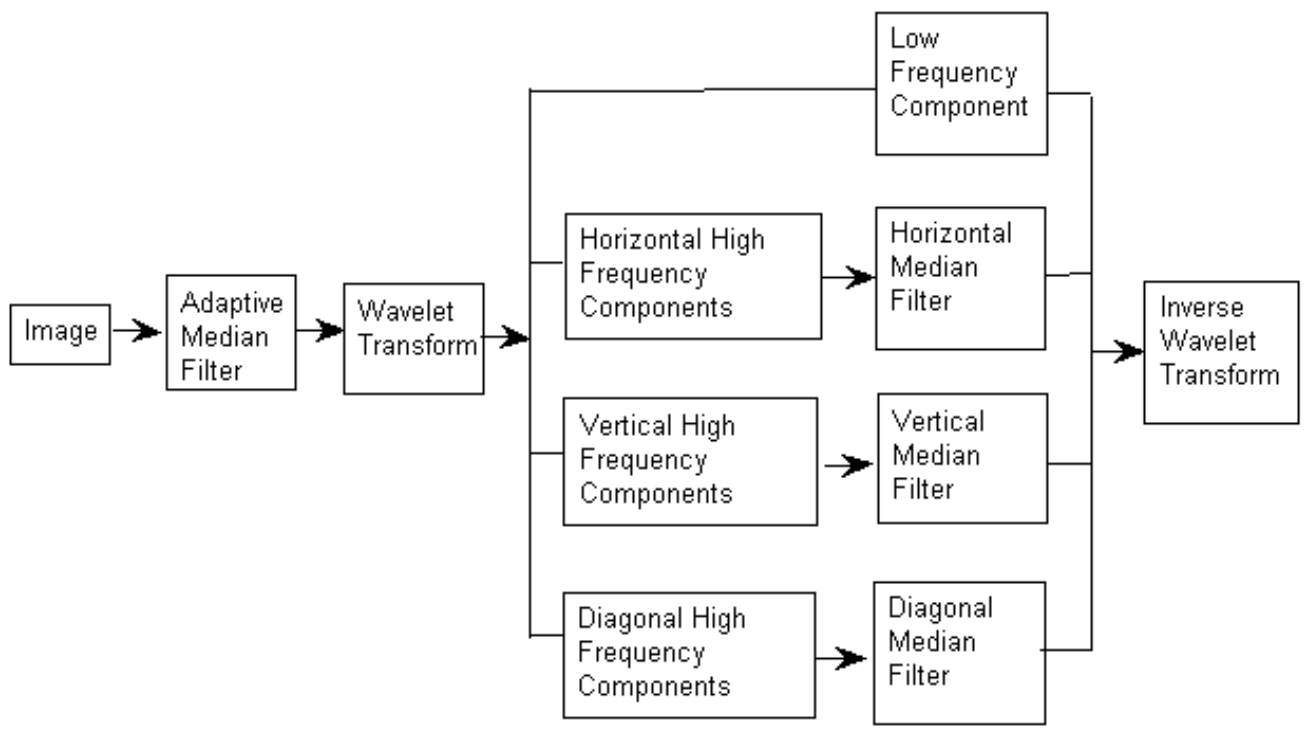

Fig. 2 denoising algorithm proposed in this paper

\section{Experimental Results and Analysis}

To prove the effectiveness of the method proposed, denoising the image in figure 3 (a) by using the method proposed in this paper, and the results are compared with adaptive median filtering, wavelet threshold denoising. Threshold denoising method uses the SURE threshold estimation method to select the threshold. Due to the good symmetry of sym wavelet, it is more suitable for image processing, and can reduce the phase shift of reconstruction. This paper uses Sym8 wavelet base for the wavelet decomposition of the image, and the wavelet decomposition series is 3 . The results are shown in Figure 4: Figure 3(a) is the results of using using adaptive median filtering. Figure 3(b) is the result of using wavelet soft threshold, figure 3(c) is the result of using the proposed method. 

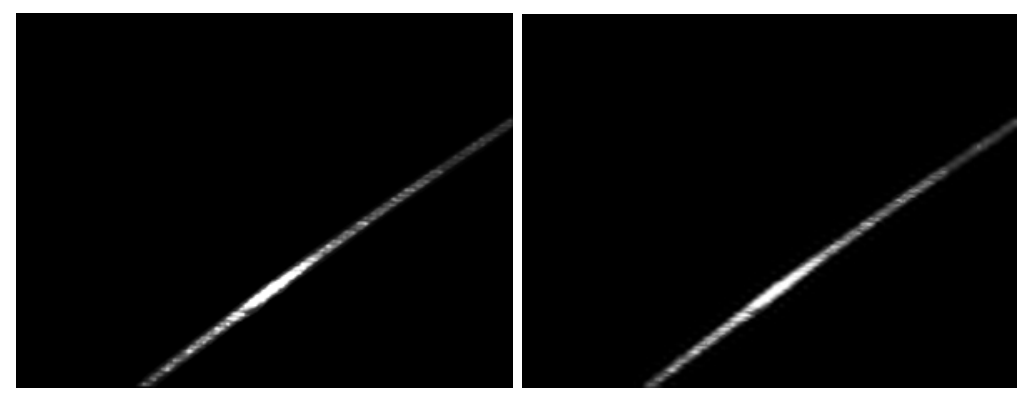

b Wavelet Soft-threshold

a Adaptive median filter

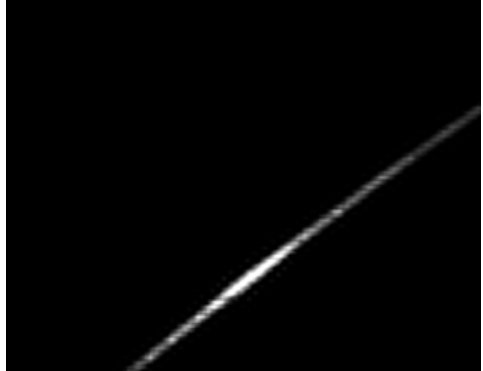

c algorithm proposed in this paper

Fig. 3 Comparison of three denoising methods

In order to objectively evaluate the denoising method proposed in this paper, we use the peak signal to noise ratio (PSNR) and the mean square error (MSE) to measure the denoising effect. The bigger the PSNR, the smaller the MSE, the better the denoising effect. The formula is shown in (1) and $(2), I^{\prime}(x, y)$ and $I(x, y)$ represent the gray value of the denoised image and the original image.

$$
\begin{gathered}
P S N R=10 \times \lg \frac{255^{2}}{\frac{1}{M \times N} \sum_{x=1}^{M} \sum_{y=1}^{N}\left[I^{\prime}(x, y)-I(x, y)\right]^{2}} \\
M S E=\frac{\sum_{x=1}^{M} \sum_{y=1}^{N}\left[I^{\prime}(x, y)-I(x, y)\right]^{2}}{M \times N}
\end{gathered}
$$

As shown in table 1 , the peak signal to noise ratio (PSNR) and the mean square error (MSE) value of the denoised image by three methods are compared. As can be seen from the table, Compared with the other two methods, the method proposed in this paper has better effect.

Table 1. Comparison of three denoising methods.

\begin{tabular}{ccc}
\hline method & PSNR(dB) & MSE \\
\hline Adaptive median filter & 32.3 & 40.7 \\
Wavelet Soft-threshold algorithm & 32.0 & 41.2 \\
algorithm proposed in this paper & 37.4 & 12.9 \\
\hline
\end{tabular}

\section{Conclusion}

In this paper, an improved image denoising algorithm based on wavelet transform and adaptive median filter is proposed. Firstly, the spatial adaptive median filter is used to construct the image, then the wavelet transform is performed, and median filter is used to filter the high frequency coefficients obtained from wavelet transform, the denoised image is obtained by wavelet reconstruction of low frequency coefficients and filtered high frequency coefficients. The experimental results show that the algorithm proposed can remove the salt and pepper noise and Gauss noise and has better effect compared with the denising method of mean filter, adaptive median filter and the traditional wavelet soft threshold.

\section{References}

[1]. He Junji., Zhang Guangjun. (2003) Study on method for processing image of strip in structured-light 3D vision measuring technique. Journal of Beijing University of Aeronautics and Astronautics, 29(7), 593-597.

[2]. Li Yingying., Zhang Zhiyi., Yuan Lin. (2013) Survey on Linear Structured Light Stripe Center Extraction. Laser \& Optoelectronics Progress, 50(10), 100002.

[3]. Lei Qiaoli. (2011) Machine Vision Measurement Technology and Applications Based on LabVIEW. Xidian University, 19-20.

[4]. Zhao Bohua, Wang Boxiong, Zhang Jin. (2011) Extraction of laser stripe center on rough metal surface. Optics and Precision, 19(9), 2138-2415. 
[5]. Rafael C. Gonzales., Richard E. Woods. (2011) Digital Image Processing, Third Edition. Ruan Qiuqi, Ruan Yuzhi Transl. Publishing House of Electronics Industry, 96, 209-210. 